\title{
PERFIL EPIDEMIOLÓGICO DOS CASOS DE LEISHMANIOSE NO MUNICÍPIO DE ITAPERUNA - RJ: DE JANEIRO DE 2006 A OUTUBRO DE 2009
}

\author{
Alair Ignácio de Oliveira ${ }^{1 *} \&$ Paula Hocayen de Paula ${ }^{1}$
}

http://dx.doi.org/10.18571/acbm.006

\section{RESUMO}

A Leishmaniose Tegumentar Americana é uma zoonose que representa um problema de saúde pública. Tal doença é causada por protozoários do gênero Leishmania e transmitida por insetos vetores pertencente a várias espécies de Flebotomíneos. Este trabalho teve objetivo de traçar um perfil epidemiológico da Leishmaniose Tegumentar Americana no município de Itaperuna, localizado a noroeste do Estado do Rio de Janeiro, Brasil. Para tanto, foi realizado o levantamento de dados nas principais unidades de saúde do município de janeiro 2006 a outubro 2009. Durante o referido período foram notificados e confirmados 05 casos em 2006 e 04 casos em 2007. No ano de 2008 não houve casos notificados. Os dados analisados até o mês de outubro 2009 revelaram apenas 01 caso notificado. As notificações foram relacionadas principalmente à pacientes de zona rural do município de Itaperuna e adjacentes. A maioria dos casos relatados ocorreu em moradores ou trabalhadores da zona rural. A visita in locu revelou a necessidade de um trabalho preventivo através de campanhas de combate ao inseto vetor.

Palavras chave: Leishmaniose, investigação epidemiológica, zoonose, zona rural.

\begin{abstract}
American Tegumentary Leishmaniasis is a zoonose that represents a public health problem. This disease is caused by Leishmania genus protozoa and transmitted by Flebotomineos insects vectors. The aim of this work was characterize an epidemiological profile of the Leishmaniasis American Tegumentary at Itaperuna city, located the northwest of the State of Rio de Janeiro, Brazil. For this, data were collected in the mains units of health on the city from January 2006 to October 2009. During this period, were notificated and confirmed 05 cases in 2006 and 04 cases in 2007. In 2008 no cases were notified. One case were notified on October 2009. The notifications were mainly related to patients from rural zone and proximities. By the end of this work is evident that the majority of notified cases occurred in inhabitants or workers of rural zone. In locu visit strongly suggest the necessity of a preventive work with campaigns to insect vector combat.
\end{abstract}

Keywords: Leishmaniasis, epidemiological investigation, zoonosis, rural zone.

1 Universidade Iguaçu - Campus V - Itaperuna

*Autor para correspondência: ignacioliveira@gmail.com 


\section{INTRODUÇÃO}

A Leishmaniose Tegumentar Americana (LTA) é uma doença parasitária da pele e mucosas, causada por protozoários de gênero Leishmania Brasil (1998). É uma zoonose de animais silvestres que atinge o homem quando este entra em contato com focos zoonóticos Guerra et al. (2007) áreas de desmatamento ou extrativismo Brasil (2004). Os protozoários parasitas, com um ciclo de vida digenético (heteroxênico), vivendo alternadamente em hospedeiros vertebrados e insetos vetores Gontijo e Carvalho (2003), onde sofre transformações em sua forma vegetativa até o amadurecimento, se tornando infectante e através da picada do vetor, passa para outro organismo suscetível (Brasil, 2006).

Há várias espécies de Leishmanias envolvidas na transmissão. No Brasil, as mais importantes são Leishmania (Viannia) braziliensis, Leishmania (Leishmania) amazonensis e Leishmania (Viannia) guyanensis Brasil (2004; 2005; 2008), que têm vários hospedeiros naturais e selvagens como: marsupiais (Dedelphis mucura), raposa (Cerdocion tolos), preguiça (Choloepus didactilus) tamanduá (Tamandua tetradactyla) cão (Canis familiaris) Barbosa, et al. (1999) cavalos, mulas, roedores, principalmente o roedor rato-soiá (Proechymis), alem do Oryzamys (Brasil, 1998; 2002; 2004; 2007; Basano; Camargo, 2004).

As leishmaniose se estendem, em nosso país, por extensas áreas das regiões Norte, Nordeste, Sudeste e Centro Oeste Borges, et al. (1999). Até a década de 1940 estava intimamente relacionada com a penetração do homem em zonas de florestas em desbravamento, com a derrubada de matas para o plantio, a construção de estradas de rodagem, Fernandes et al. (2004) ferrovias, hidrelétricas e implantação de povoados (Costa et al., 2004).

Essas alterações, de origem antrópica, Guerra, et al. (2007), estão mudando o perfil epidemiológico da leishimaniose que antes era mantida predominantemente em um ciclo silvestre (Costa et al., 2004).

Hoje, com a transmissão ocorrendo em periferias de área urbana, e em ambientes domiciliares e peridomiciliares atinge também crianças e mulheres, (Guerra, et al., 2007; Lima, et al., 2002; Lessa, et al., 2007).

As infecções causam um largo espectro de alteração clínicas que dividem a leishmaniose em quatro amplas categorias, baseadas na extensão e na gravidade do acontecimento hospedeiro humano, que são: leishmaniose cutânea (LC), leishmaniose cutânea difusa (LTD), e a leishmaniose mucocutânea (LMC) e a mais grave que é a leishmaniose visceral (LV), Klaus e Frankenburg (2004). Aproximadamente 350 milhões de indivíduos vivem em áreas de transmissão das formas cutâneas e cutaneomucosas e 500 mil da forma visceral (Lima, et al., 2002).

No Brasil a LTA tem caráter endêmico e está distribuída em todos os estados. A doença abrange desde formas inaparentes, ou seja, lesões discretas de pele que podem evoluir espontaneamente para cura ou ulcerações múltiplas, lesões de mucosas até formas com tendência as, metástases e recidivas, de curso lento e tratamento (Silveira, et al., 1996; Brasil, 2006).

Esta pesquisa foi desenvolvida em Itaperuna, que é um Município de grande importância para o Noroeste Fluminense, sendo referência no turismo, agricultura, educação e saúde. Na saúde recebe pacientes de várias partes do estado e principalmente dos municípios vizinhos.

O estudo, de natureza quantitativa e de cunho bibliográfico, teve como objetivo fazer um levantamento epidemiológico dos casos notificados de LTA, no município, de janeiro de 2006 a outubro de 2009, com o intuito de traçar um perfil da doença neste município. Através deste perfil, medidas eficientes e programadas poderão ser tomadas, visando à melhoria da 
condição de vida da população de Itaperuna em geral e principalmente da parcela mais susceptível a LTA.

\section{MATERIAL E MÉTODO}

Esta pesquisa foi desenvolvida no município de Itaperuna, com dados obtidos no período de janeiro de 2006 a outubro de 2009. Este Município tem grande importância para o Estado do Rio de Janeiro, sendo referência no turismo, agricultura, educação e saúde. Na saúde recebe pacientes de várias partes do estado e principalmente dos municípios vizinhos.

Localiza-se no Noroeste do Estado do Rio de Janeiro, ocupando uma área de 1.106,4 $\mathrm{Km}^{2}$. Apresenta uma população residente de 99.454 habitantes, (IBGE, 2009). Clima quente, fauna rica em animais silvestres.

Foi feito o levantamento de dados no banco de sangue e ambulatório do Hospital São José do Havaí, no laboratório e ambulatório do Centro de Saúde Raul Travassos (C S R T) e no serviço de Epidemiologia da Secretaria Municipal de Itaperuna como forma de levantar dados para este estudo.

Estes dados dizem a respeito à pacientes de Itaperuna e região, que procuram o Hospital São José do Avaí (H.S.J.A.) ou o Centro de Saúde Raul Travassos (C.S.R.T.).

e quando apresentam sintomas da LTA são encaminhados ao serviço de Epidemiologia da Secretaria de Saúde de Itaperuna.

O diagnóstico da doença em Itaperuna foi baseado nos critérios clínicos e epidemiológicos, submetidos à biópsia de pele ou mucosa, teste de Montenegro e exames de cultura com detecção do parasito nas lesões.

\section{RESULTADOS}

Durante a pesquisa foi feito o levantamento das 16 fichas de pacientes, que por residirem em área com casos já confirmados de LTA e por apresentarem sintomas similares, foram notificados no serviço de Epidemiologia de Itaperuna. Dos pacientes notificados, depois de realizados os exames laboratoriais ficaram comprovados que haviam 10 casos autóctones confirmados, do período de janeiro de 2006 a outubro de 2009.

No ano de 2006, 05 casos foram confirmados e os dados referentes a eles estão listados, na Tabela 01.

Observa-se na tabela 01 que dos 05 casos 03 casos ocorreram em Valão do Cágado, uma localidade que pertence ao município de Itaperuna. Os outros 02 casos ocorreram em Laje do Muriaé, município próximo a Itaperuna. Dos 05 pacientes, 03 são do sexo masculino e 02 do sexo feminino, com idades variadas. Houve cura de 02 pacientes neste período.

Já no período de janeiro 2007 a outubro de 2009, foram confirmados 05 casos, listados na Tabela 02:

\section{Tabela 02: Casos confirmados de Leishimaniose (LTA) no período de janeiro de 2007 a outubro de 2009.}

Observa-se na tabela 02 a ocorrência de 01 caso de paciente oriundo do município de Bom Jesus do Itabapoana, sendo que o mesmo reside na zona rural deste município, muito próxima da localidade do Valão do Cágado. Nota-se também a reincidência dos 03 casos de pacientes que residem na mesma localidade. Dos 05 pacientes 03 são do sexo masculino e 02 do sexo feminino, com idades variadas. 
Em 2008 não foram relatados casos e em 2009 até o mês de outubro, só foi notificado apenas 01 caso, sendo paciente oriundo do município de Porciúncula município este próximo a Itaperuna, dados sobre cura não foram publicados.

Notam-se pelos dados de 2006 e 2009, que os pacientes notificados não eram todos do Município. Estes pacientes, oriundos de outros municípios, muitas vezes não conseguem realizar os exames confirmatórios em seus municípios e procuram, ou são encaminhados pelas unidades hospitalares dos mesmos para o Hospital São José do Avay. Quando confirmados são encaminhados para o serviço Epidemiológico da Secretaria Municipal de Itaperuna.

Foi constatado também que nenhuma outra unidade hospitalar do município, encaminhou paciente para o serviço de epidemiologia da secretaria municipal de saúde de Itaperuna.

De 2002 a 2006 só há confirmação de casos isolados, sem a localidade, listados na Tabela 03.

\section{DISCUSSÃO}

Tanto Itaperuna quanto Laje do Muriaé e Bom Jesus do Itabapoana são municípios que se situam em área de relevo, recobertos por remanescentes de Mata Atlântica, aonde vem acontecendo ao longo dos anos um processo progressivo de ocupação humana, associado a um crescente desmatamento, causando alterações ambientais. Com estas alterações vem sendo modificando o perfil epidemiológico da leishmaniose em áreas onde a Leishmania era mantida predominantemente silvestre. As alterações ambientais proporcionam a adaptação do flebotomíneo ao ambiente peridomiciliar ou até mesmo domiciliar, ocorrendo à transmissão da Leishmania a animais domésticos e ao homem.

É demonstrado no presente trabalho que os 03 casos confirmados em Itaperuna, são de pacientes da localidade do Valão do Cágado. Esta comunidade se localiza na zona rural de Itaperuna, região agrícola, que se caracterizam por encostas, pequenas matas (arvoredos), com residências muito próximas as matas.

O paciente oriundo de Bom Jesus do Itabapoana reside em zona rural com as mesmas características da região citada e bem próxima a ela. Mostra também que 09 pacientes moram na zona rural ou são trabalhadores rurais.

Situação parecida foi encontrada por Vanzeli e Kanamura, (2007) em Ubatuba, São Paulo (Brasil). Analisando casos confirmados no período de 1994 a 2004, observaram que a maioria deles ocorreu em locais onde as residências eram construídas de forma desordenadas, entre 50 e 10 metros de distância das matas, onde $86 \%$ dos casos encontrados eram nas residências mais próximas a ela. Situação parecida também foi descrito por Silveira et al., (1996), com pesquisa realizada do período de 1992 a 1993 em área endêmica, em propriedades da zona rural nos Municípios de Jussara e Terra Nova, no Norte do Estado do Paraná, (Brasil). Os casos registrados da localidade de Valão do Cágado, com reincidência dos 03 pacientes que foram notificados no ano de 2006 e de novo no ano de 2007, mostram que esta é uma área endêmica do município.

Estes dados mostram que o ambiente modificado pela ação do homem com atividades agrícolas diversas, formação de pastagem restando hoje poucas florestas naturais, proporciona a disseminação da doença. Isto prova que o homem cada vez mais está invadindo o habitat natural do inseto, sendo, portanto, mais propenso a ter contato com o vetor e sendo infectado. Como descreveram Sessa, et al. (1994), em investigação feita nos municípios de Santa Leopoldina e Afonso Cláudio, no Estado do Espírito Santo (Brasil) onde afirma que a Leishmaniose (LTA) tem sido registrada em áreas de colonização antiga, Lima, et al. (2007), em levantamento feito em 11 municípios do Paraná, com sede no Município de Cianorte, 
onde constatou que a leishmaniose tegumentar tem distribuição geográfica ampla e irregular, coincidindo com áreas de alto grau de destruição da vegetação nativa, em decorrência da exploração agrícola por monoculturas.

Do total de 09 pacientes, 05 eram do sexo masculino. Apesar de ser uma pequena diferença, este dado mostra que o homem tem mais contato com o local onde o vetor se desenvolve. Fato que também foi observado por Lima, et al. (2002), na pesquisa realizada no estado do Paraná, no período de 1993 a 1998,onde verificou-se que dentre os 4.416 casos $2.808(63,6 \%)$ eram do sexo masculino, fato também comprovado por Naiff Junior et al. (2009), em pesquisa realizada no Município de Rio Preto da Eva AM. No período de abril de 2006 a março de 2007dos 113 foram confirmados, 77 (68,1\%) eram do sexo masculino, resultado também encontrado por, Freitas et al. (2006) em pesquisa realizada no município de Jussara, no Estado do Paraná (Brasil) onde 84,5\% do pacientes eram do sexo masculino. Além disso, a maioria dos homens são trabalhadores rurais, com mais acesso ao criadouro do vetor, como descrito por Silveira, et al. (1996) quando realizou pesquisa na área rural do município de Jussara e Terra Boa, norte do Estado do Paraná (Brasil) onde constatou que de 136 casos positivos do município de Jussara, $75,7 \%$ eram do sexo masculino trabalhadores rurais. Observa-se também que as idades variam, com pacientes de 18 anos até 59 anos, mostrando que qualquer pessoa é susceptível, desde que tenha contato com o vetor. Fato também observado por Chagas, et al. (2006) em pesquisa feita na Vila de Pitanga no município de Presidente Figueiredo- AM, onde de 56 casos, 86,2\% eram do sexo masculino na faixa etária entre 15 a 45 anos. Esta situação é descrito também por Sessa, et al. (1994), no período de 1991 a 1993. Os autores desenvolveram pesquisa nos municípios de Afonso Cláudio e Santa Leopoldina no Estado do Espírito Santo (Brasil), e citam que a idade não tem influencia na transmissão da doença.

Os casos confirmados começaram a surgir no ano de 2000, sendo que a partir desse ano até 2005 surgiram outros casos isolados. O caso de 2005 foi o primeiro a ser descrito na localidade do Valão do Cágado, que hoje apresenta 03 casos reincidentes. Isto mostra que não há controle do vetor nem a orientação aos moradores daquela localidade, no sentido de se prevenir, pelo serviço de Epidemiologia da Secretaria Municipal de Itaperuna. A prevenção é imprescindível para o controle da LTA, fato comprovado por Costa, et al. (2004) em pesquisa realizada em PARATY-RJ, no período 2002 a 2003, onde foi feito um trabalho de controle e prevenção no município. Com este trabalho de prevenção, houve uma diminuição de $31,6 \%$, dos casos da doença, comparando-se com os dados de 2002.

É possível haver outros doentes na localidade do Valão do Cágado que não foram descritos por causa de desinformação. Os pacientes que residem nesta localidade são pessoas simplórias, sem escolaridade que não têm acesso a essas informações e nem sempre a saúde pública está atenta a elas.

Segundo o serviço de Epidemiologia da Secretaria Municipal de Saúde de Itaperuna, foi iniciado no ano de 2008, um programa de controle da LTA, de acordo com a metodologia proposta pela Fundação Nacional de Saúde (FUNASA) do manual de controle da LTA de 2000, adaptados a peculiaridades locais. O conjunto de medidas adotadas baseou-se na captura do vetor (flebotomíneos) em vários domicílios do município.

Trabalhos preventivos parecidos foram feitos na cidade de Paraty (RJ) descrito por Costa et al. (2004), e no conjunto residencial Inocente Vila Nova Júnior no município de Maringá, (PR), descrito por Carfan, et al. (2004) mostrando resultados bastante satisfatórios com redução dos casos de LTA em 31,6\% (Paraty), 82,5\% em Maringá.

Quando o trabalho de levantamento de dados foi iniciado, percebeu-se o quanto era precário o sistema de informações da Epidemiologia no que diz respeito às leishmanioses. Mas ao mesmo tempo isto serviu de estímulo, pois se estava fazendo algo útil e necessário não só para os pacientes LTA positivos, como também para os que lidam no dia-dia com a 
doença: Médicos, Farmacêuticos, Enfermeiros e outros Profissionais envolvidos, familiares. Acredita-se que o presente trabalho tenha atingido os profissionais envolvidos com a leishmaniose, pois foi feito à atualização das fichas epidemiológicas, mostrando uma mudança.

\section{CONCLUSÃO}

Foram notificados 10 casos de LTA em Itaperuna de janeiro de 2006 a outubro de 2009, sendo que as maiorias dos casos são da localidade de Valão do Cágado, inclusive com reincidência de 03 pacientes de 2006 em 2007.

$\mathrm{O}$ acesso ao ambiente, degradado pelo homem e criadouro do vetor é determinante na transmissão da doença.

É importante ressaltar que além das medidas de controle especificas, é necessário o esclarecimento da população quanto às formas de prevenção, transmissão, tratamento da leishmaniose. Deve-se também proporcionar a produção e a utilização de conhecimentos que serem implantados ou implementados, tendo como objetivo garantir os princípios e diretrizes do SUS, e a saúde para o desenvolvimento do ser humano que resgata o vinculo de corresponsabilidade entre o serviço público e a população. A saúde pública destes municípios não desenvolve programas eficientes e sistemáticos para a erradicação desta doença.

Desta forma encontra-se neste contexto relevância científica e social, bem como justificativa para a investigação que ora se propõe.

\section{REFERENCIAS}

BARBOSA, G. S.; MARZOCH, M. C. A. MASSARD, C. L. LIMA, G. P. S.; CONFORT, E. M. Aspectos epidemiológicos da leishmaniose tegumentar americana em cães, no Município de Paraty, Estado do Rio de Janeiro, Brasil, Cad. Saúde Pública vol.15, p. 641-646, Rio de Janeiro July/Set. 1999.

BASANO, S. A.; CAMARGO, L. M. A. Leishmaniose tegumentar americana: histórico, epidemiologia e perspectivas de controle. Revista Brasileira de Epidemiologia, v.7, p. 328-37, 2004.

BORGES, A. S.; MACHADO, A. A.; FERREIRA, M. S.; FIGUEREDO, J. F. C.; CIMERMAN, S.; BACHA, H. A. Concomitância de Leishmaniose e infecção pelo vírus da imunodeficiência humana (HIV); estudo de casos, Revista da Sociedade Brasileira de Medicina Tropical, v. 32, p. 713-719, 1999.

BRASIL. Ministério da Saúde. Doenças Infecciosas e Parasitárias. 4 ed. Ampliada, Brasília, DF: MS, p.208-215, nov. 2004

BRASIL. Ministério da Saúde. Doenças Infecciosas e Parasitárias. Guia de Bolso $5^{\text {a }}$ ed. Ampliada. Brasília; 2005.

BRASIL. Ministério da Saúde. Doenças Infecciosas e Parasitárias. 6 ed. Brasília, DF: MS, p.196-199, 2006.

BRASIL. Ministério da Saúde. Doenças Infecciosas e Parasitárias. Guia de Bolso $7^{a}$ ed. revista, Brasília; 2008. 
BRASIL. Ministério da Saúde. Guia de vigilância epidemiológica. Fundação Nacional de Saúde. Brasília; 1998.

BRASIL. Ministério da Saúde. Guia de vigilância epidemiológica/ Fundação Nacional de Saúde, v.2, 5 ed. Brasília, DF: FUNASA, 2002, p. 501-524.

BRASIL. Ministério da Saúde. Manual de Vigilância da Leishmaniose Tegumentar Americana. 2. edição atualizada 2007, p. 7-179.

BRASIL. Ministério da Saúde. Manual de Vigilância e Controle da Leishmaniose Visceral. 1 ed. $3^{\text {a }}$ reimpressão Brasília, DF: MS, 2006p. 37-39.

CARFAN, C.; ANGELIS, B. L. D.; MENEGUETTI, C.; OLIVEIRA, M. C.; PEREHOUSKEI, N. A.; ICHIBA, S.H. K. Leishmaniose Tegumentar Americana: o caso do conjunto residencial Inocente Vila Nova Júnior no município de Maringá, Estado do Paraná, 2001-2004, Acta Scientiarum HECTH, Maringá. 26, p. 341-344, 2004.

CHAGAS, A. C.; PESSOA, F. A. C.; MEDEIROS, J. F.; DANIE,L V. P.; MESQUITA, E. C.; BALESTRASSI, D. A. Leishmaniose Tegumentar Americana (LTA) em uma Vila de Exploração de Minérios- Pitanga Município de Presidente Figueiredo, Amazonas, Brasil, Revista Brasileira Epidemiológica v. 9, p. 186-192, 2006.

COSTA, C. M.; MOUTINHO, F. F.; BRUNO, S. F. A. Experiência do Município de Paraty (Rio de Janeiro, Brasil) na prevenção e controle da leishmaniose tegumentar americana. Caderno de Saúde Publica v.59, p. 110-14, 2004.

FERNANDES, N. C.; ISABMORGAN, I.; MACIERA J. P.; CUZZI T.; NOE R. A. M., Leishmaniose tegumentar americana: casuística hospitalar no Rio de Janeiro: investigação clínica, laboratorial e terapêutica, Anal Brasileiro de Dermatologia. V.79, 2004.

FREITAS, J.S.; SANTANA, R.G.; MELO, S. R. Levantamento dos casos de leishmaniose registrados no município de Jussara, Paraná, Brasil. Arq. Ciênc. Saúde Unipar, Umuarama, v. 10, p. 23-27, jan./abr., 2006.

GONTIJO, B.; CARVALHO, M. L. R. Leishmaniose Tegumentar Americana Revista da Sociedade Brasileira de Medicina Tropical, v.36, p.71-80, 2003.

GUERRA, J. A. O.; BARBOSA, M. G. V.; LOURERO, A. C. S. P.; COELHO, C. P.; ROSA, G. G.; COELHO, L. I. A. C. R. Leishmaniose tegumentar americana em crianças: aspectos epidemiológicos de casos atendidos em Manaus, Amazonas, Brasil, Caderno de Saúde Pública v.23, p. 2215-2223, 2007.

IBGE, Instituto Brasileiro de Geografia e Estatísticas, Disponível em <http://www.ibge.gov.br/cidadesat/topwindow.htm?1 > Acesso em: 15 de dez. 2010.

KLAUS, S.N.; FRANKENBURG, S. Leishmaniose e outras infecções por Protozoários. In: FITZPATRICK, T.B.,FREDBERG, I.M.; EISEN, A. Z.; WOLFF, K.; AUSTEN, K. F.;GOLDSMITH, L. A.; KATZ, S. Infestação: Leishmaniose e Outras Infecções por protozoários, Tratado de Dermatologia .Rio de Janeiro-RJ: ed. Revinter, 2004. p. 2609-2615.

LESSA, M. M.; LESSA, H. A.; CASTRO, T. W. N.; OLIVEIRA, A.; SCHERIFER, A.; MACHADO, P.; CARVALHO, E. Leishmaniose mucosa: aspectos clínicos e epidemiológicos, Revista Brasileira Otorrinolaringologia v.73, São Paulo Nov./Dec. 2007. 
LIMA, A. P.; MINELli, L.; TEODORO, U.; COMUNELLO, E. Distribuição da Leishmaniose Tegumentar por imagens de sensoriamento remoto orbital, no Estado do Paraná, Brasil, investigação clínica, laboratorial e terapêutica, Anal Brasileiro de Dermatologia. v.77, p. 681-692, 2002.

LIMA, M. V. N.; OLIVEIRA, R. Z.; LIMA, A. P.; FELIX, M. L. O.; SILVEIRA, T. G. V.; ROSSI, R. M.; TEODORO, U. Atendimento de pacientes com leishmaniose tegumentar americana: avaliação nos serviços de saúde de municípios do noroeste do Estado do Paraná, Brasil, Revista da Saúde Pública, v. 23, n. 12, p. 2938-2948, dez, 2007.

NAIFF JUNIOR, R. D.; PINHEIRO, F. G.; NAIFF, M. F. SOUZA, I. S.; CASTRO, L. M. MENEZES, M. P, FRANCO A. M. R. Estudo de uma série de casos de Leishmaniose Tegumentar Americana no Município de Rio Preto da Eva Amazonas, Brasil, Revista de Patologia Tropical, v.38, p. 103-115, 2009.

SESSA, P. A.; FALQUETO, A.; VAREJÃO, J. B. Tentativa de Controle da Leishmaniose Tegumentar Americana por Meio de Tratamento dos Cães Doentes. Caderno de Saúde Publica v.10, p.457-463, 1994.

SILVEIRA, T. G. V.; UESLEI, T.; LONARDONI, M. V. C.; GUILHERME, A. L. F.; TOLEDO, M. J. O.; RAMOS, M.; ARRAES, S. M. A. A.; BERTOLINI, D. A.; SPINOZA, R. P.; BARBOSA, O. C. Aspectos Epidemiológicos da Leishmaniose Tegumentar em Área Endêmica do Estado do Paraná, Brasil. Caderno Saúde Pública, v.12, p. 141-147, 1996.

VANZELI, A.C.; KANAMURA H.Y. Estudos de fatores socioambientais associados à ocorrência de leishmaniose tegumentar americana no município de Ubatuba, SP: Brasil. Revista Panamericana de Infectologia, v.9, p. 20-25, 2007. 
Tabela 1: Casos confirmados de Leishmaniose (LTA) no período de janeiro a Dezembro de 2006, em Itaperuna.

\begin{tabular}{|c|c|c|c|c|c|c|c|}
\hline $\begin{array}{c}\text { Casos } \\
\text { Confirmados } \\
\end{array}$ & Localidade & Sexo & Idade & Profissão & $\begin{array}{l}\text { Zona } \\
\text { rural }\end{array}$ & Cura & Reincidente \\
\hline Paciente 01 & $\begin{array}{l}\text { Laje do } \\
\text { Muriaé }\end{array}$ & $\mathrm{M}$ & 58 & $\begin{array}{l}\text { Técnico } \\
\text { eletrônico }\end{array}$ & Não & Sim & Não \\
\hline Paciente 02 & $\begin{array}{l}\text { Valão do } \\
\text { Cágado }\end{array}$ & M & 24 & Lavrador & Sim & Não & Não \\
\hline Paciente 03 & $\begin{array}{l}\text { Valão do } \\
\text { Cágado }\end{array}$ & $\mathrm{F}$ & 59 & $\begin{array}{l}\text { Dona de } \\
\text { casa }\end{array}$ & Sim & Não & Não \\
\hline Paciente 04 & $\begin{array}{l}\text { Valão do } \\
\text { Cágado }\end{array}$ & $\mathrm{F}$ & 53 & $\begin{array}{l}\text { Dona de } \\
\text { casa }\end{array}$ & Sim & Não & Não \\
\hline Paciente 05 & $\begin{array}{l}\text { Laje do } \\
\text { Muriaé }\end{array}$ & M & 38 & Lavrador & Sim & Sim & Não \\
\hline
\end{tabular}


Tabela 02: Casos confirmados de Leishimaniose (LTA) no período de janeiro de 2007 a outubro de 2009.

\begin{tabular}{|c|c|c|c|c|c|c|}
\hline $\begin{array}{c}\text { Casos } \\
\text { Confirmados }\end{array}$ & Localidade & Sexo & Idade & Profissão & $\begin{array}{l}\text { Zona } \\
\text { rural }\end{array}$ & Reincidente \\
\hline Paciente 01 & $\begin{array}{l}\text { Valão do } \\
\text { Cágado }\end{array}$ & $\mathrm{F}$ & 59 & $\begin{array}{c}\text { Dona de } \\
\text { casa }\end{array}$ & Sim & Sim \\
\hline Paciente 02 & $\begin{array}{l}\text { Valão do } \\
\text { Cágado }\end{array}$ & M & 24 & Retireiro & Sim & Sim \\
\hline Paciente 03 & $\begin{array}{l}\text { Valão do } \\
\text { Cágado }\end{array}$ & $\mathrm{F}$ & 53 & $\begin{array}{l}\text { Dona de } \\
\text { casa }\end{array}$ & Sim & Sim \\
\hline Paciente 04 & $\begin{array}{l}\text { Bom Jesus do } \\
\text { Itabapoana }\end{array}$ & M & 18 & Lavrador & Sim & Não \\
\hline Paciente 05 & Porciúncula & M & 12 & Menor & Sim & Não \\
\hline
\end{tabular}

$\mathbf{F}=$ Feminino $; \mathbf{M}=$ Masculino 
Tabela 03: Casos confirmados de Leishmaniose (LTA), no período de 2000 a 2006.

\begin{tabular}{ccccccccc}
\hline Ano & $\mathbf{2 0 0 0}$ & $\mathbf{2 0 0 1}$ & $\mathbf{2 0 0 2}$ & $\mathbf{2 0 0 3}$ & $\mathbf{2 0 0 4}$ & $\mathbf{2 0 0 5}$ & $\mathbf{2 0 0 6}$ & Total \\
\hline Casos & 1 & 1 & 3 & 2 & 1 & 1 & 5 & 14 \\
Cura & 0 & 1 & 3 & 2 & 1 & 1 & 4 & 12 \\
Transf. & 1 & 0 & 0 & 0 & 0 & 0 & 0 & 1 \\
\hline \hline
\end{tabular}

